\title{
REVIEW ON CUBOSOMES
}

\author{
SARITHA M. ${ }^{1}$, BOYINA HARSHINI ${ }^{*}$, P. V. KAMALA KUMARI ${ }^{3}$, Y. SRINIVASA RAO ${ }^{4}$ \\ Department of Pharmaceutics, Vignan Institute of Pharmaceutical Technology, Duvvada, Visakhapatnam, 530049 \\ Email: chsaritha1975@gmail.com
}

Received: 06 Aug 2021, Revised and Accepted: 10 Oct 2021

\begin{abstract}
Cubosomes are stable nanostructured liquid crystalline particles which are made of a specific group of amphiphilic lipids in definite proper ratio in water and then stabilised by biocompatible substances like triblock polymer. Cubosomes are curved bicontinuous cubic phase liquid crystals and they can split to form thermodynamically stable particulate dispersions. Cubosomes have biocompatible and bio-adhesive properties andare capable of loading 3D bilayered structure resembling honeycomb (carvenous) like structure by encapsulating lipophilic, hydrophobic and amphiphilic substances. Cubosomes are administered through different ways such as orally, parenterally and percutaneously. Cubosomes are versatile systems in their structure for drug delivery systems.
\end{abstract}

Keywords: Cubosomes, Drug-loading hydrophilic, Hydrophobic, Amphiphilic, Lyotropic liquid crystals, Biocontinuous, Nanoparticles, Honeycomb, Self-assembly, Anti-cancer

(C) 2021 The Authors. Published by Innovare Academic Sciences Pvt Ltd. This is an open-access article under the CC BY license (https://creativecommons.org/licenses/by/4.0/) DOI: https://dx.doi.org/10.22159/ijcpr.2021v13i6.1926 Journal homepage: https://innovareacademics.in/journals/index.php/ijcpr

\section{INTRODUCTION}

Cubosomes contains larger surface area and same microstructure as parent cubic phase and the prepared cubosomal dispersions have much lower viscosity compared to bulk cubic phase. Cubosomes were first proposed by Larsson, which reflects the cubic molecular crystallography and similar to liposomes. Selected lipids, surfactants and the polymer molecules have both polar and non-polar components i. e; amphiphilic [1]. The amphiphilic molecules follow the hydrophobic effect into polar solvent to identify and assemble into a liquid crystal of nanometre scale. Cubosomes have a great role in nanodrug formulations and are formations of bicontinuous cubic liquid crystalline phase by hydrating mixture of monoolein and poloxamer 407 [2]. In cubosomes active chemical constituent molecules are coordinated through chemical bonds to the polar head of the phospholipids. The polymer and the individual drug compound form a $1: 1$ or $2: 1$ complex ratio depending on the substance [3].

A drug delivery system is a device and formulation which safely brings a therapeutic agent to the specific body site at a specific rate to achieve an effective concentration at the of drug action. The release of the drug in a pre-designed manner is termed for controlled release (CR) to promote therapeutic benefits by minimizing toxic side effects. Sustained release of a drug over an extended period of time may reduce the need of multiple dosing which will is a benefit in terms of reduced cost and increased patient compliance. The release rate of the drugs must to be controlled to achieve optimal drug release profiles. These systems consist of liquid crystalline aggregates, which are loaded, stabilized and then deliver the active ingredients. The drugs incorporated into the complex internal domain structures that facilitates the diffusioncontrolled release of drug into the surrounding external aqueous environment $[3,4]$.

\section{Cubosomes}

The word 'Cubosomes' is derived by their structure i. e; 'phases' suffixed as 'some' and they have cubic crystal lattice, called as cubosomes. Cubosomes are bicontinuous cubic phase liquid crystals which have non-intersecting hydrophilic regions separated by a lipid bilayer that is twisted into a periodic minimal surface with zero curvature; hence they are called as 'viscous isotropic phases' [5]. Larsson was examined the structure of aqueous monoglyceride cubic phases by using X-raydiffraction, and NMR and then found the cubosomes have continuous regions of both hydrophobic and hydrophilic nature, which leads to a conclusion that the cubic phase structures explained by the concept of differential geometry and periodic minimal surfaces [6]. Cubosomes are nanoparticles which are more accurately nanostructured particles and self-assembled liquid crystalline particles with solid-like rheology [7].

Cubosomes follows the geometric model and it was supplied, prepared and examined for drug delivery. The particles of cubosomes are initially formed by the mechanical fragmentation of the lipid-water cubic phase in a three-cubic phase region that contains a liposome dispersion and differentiate from liposomes; these particles are termed as cubosomes. The structure of cubosomes maintains the efficacy and stability of activity like vitamins and proteins. Cubosomes which contains a sufficient average degree of molecular orientation in order to characterized by the structural symmetry and also form in aqueous surfactant system at a relatively highconcentrationof amphiphile [8].

\section{Structure of cubosomes}

The basic structure of cubosomes consists of a honey-combed (carvenous) structures separating the two internal aqueous channels and a large interfacial area [9]. Cubosomes are nanoparticle sized ranges from 10-500 $\mathrm{nm}$ in diameter and are likely to be spherical which appears like dots and more accurately nanostructured particles of liquid crystalline phases by a cubic crystallographic symmetry and then formed by the self-assembly of amphiphilic or surfactant like molecules [10]. Cubosomes have the amphiphilic carrier system that has the capability to encapsulate both hydrophilic and lipophilic drugs. The hydrophilic drug which is encapsulated inside the vesicles whereas the lipophilic drug is partitioned between the hydrophilic domains [11]. Cubosomes, which forms bicontinuous water and oil channels as they are Amphiphilic molecules, whereas 'bicontinuous' referred as two distinct (continuous, but non-intersecting) hydrophilic regions that are separated by the bilayer. The interconnections of the structure results in a clear, viscous gel which are similar in appearance and rheology to be cross-linked polymer hydrogels as shown in fig. 1 [12].

\section{Types of cubosomes}

\section{Liquid cubosome precursors}

The hydrotropic dilution process is meant to be produce more stable and smaller cubosomes. The Nucleation process which allows the formation ofparticles whose growth is seen under precipitation and crystallization processes [13]. 


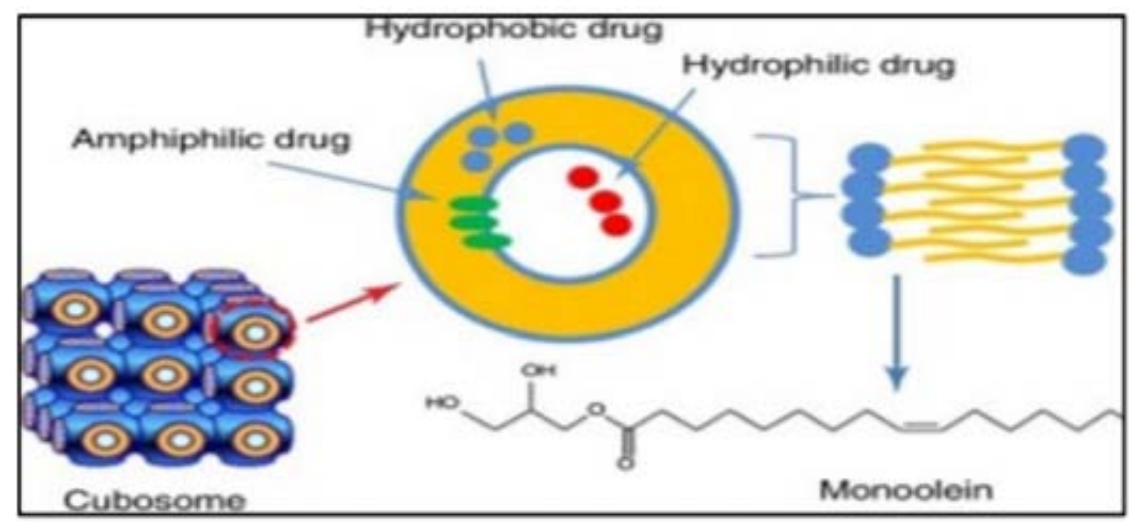

Fig. 1: Structure of cubosomes

\section{Powdered cubosome precursors}

These are composed of dehydrated surfactant coated with a polymer. These powders offer some advantages to liquid phase hydrotropic cubosome precursors. Cubosomes which are having a mean particle size of $600 \mathrm{~nm}$ are formed by the hydration of the precursor powders, which is confirmed by light scattering and cryoTEM [14]

\section{Components of cubosomes}

The components of cubosomes composed of mainly three major components Amphiphilic lipids, stabilizer, and water. The amphiphilic lipids upon hydration process produce the cubic liquid crystalline phases, whereas the stabilizers are polymeric substances which prevent the reconstitution to bulk cubic phase [15]

\section{Amphiphilic lipids}

The mainly used amphiphilic lipids are glyceryl monooleate GMO to prepare cubosomes that is usually discussed to as monoolein and phytantriol (PHYT). GMO consisting the glycerides of oleic acid and some other fatty acids mixtures, that consist mostly of monooleates which fits to the amphiphilic lipids group with a capacity to form various lyotropic liquid crystals as shown in fig. 2. GMO, that have hydrocarbon chain length in between the range of 12-22 have greater tendency to form cubic phases $[16,17]$. Furthermore, it is a biocompatible and biodegradable material generally recognized as safe (GRAS) category by FDA, which is mainly used as emulsifier in the food industry. PHYT is a chemical material having a phytanyl chain exhibits the formation of cubic phases by increasing the temperature and water content. PHYT, chemically 3,7,11,15tetramethyl-1,2,3-hexadecanetriol, is a mainly used component in cosmetic products $[18,19]$.

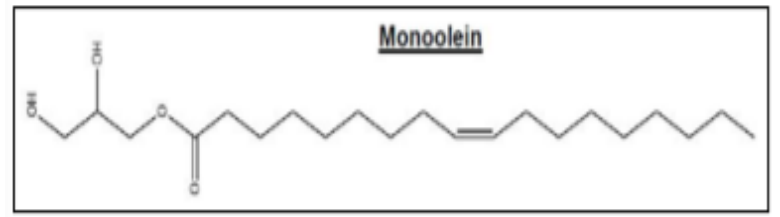

Fig. 2: Structure of glyceryl monooleate

\section{Stabilisers}

The scientists in research suggested a vital role for the surfactants as the stabilisers used to improve the stability of cubosomes against the coalescence of the bulk cubic phase. Poloxamer 407 (P407), a PEO-PPO-PEO,a tri-block copolymer which is mostly used surfactant in the preparation of cubosomes with its definite PPO portions which are being located either at the surface of the cubosomes or within the bilayer structure and the PEO chains are exposed to the surrounding water phase as shown in fig. 3 [20]. P407 is generally applied up to a concentration of $20 \% \mathrm{w} / \mathrm{w}$ depending on the quantity of dispersed phase. Wadsten-Hindrichsen investigated about the effect of three water-miscible solvents propylene glycol (PG), polyethylene glycol 400 (PEG400) and 2-meth-yl-2, 4pentanediol (MPD) on a phytosome based cubosomal system. It was discovered that the poly (ethylene oxide) stearate stabilizers were more effective than steric stabilizers in the cubosomes [21, 22].

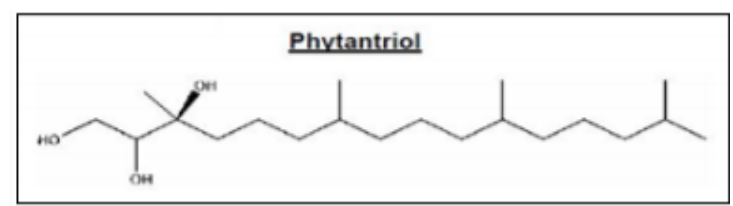

Fig. 3: Structure of phytantriol

\section{Advantages of cubosomes [23-25]}

- The cubosomes have the ability of encapsulating hydrophobic, hydrophilic and amphiphilic substances and being thermodynamically stable for longer.

- They can achieve sustained and targeted release profiles for drugs delivery.

- They are economic and non-toxic in nature.

- They have biocompatibility and bio-adhesivity properties.

- Due to high internal surface area and cubic crystalline structures, there is a high drug loading capacity.

- It involves the simple method of preparation and possess lipid biodegradability.

- Cubosomes acts as excellent solubilizers and are used in the treatment of skin, hair and other body tissues.

- Cubosomes of bicontinuous liquid cubic crystalline phases are even stable in excess water.

\section{Disadvantages}

- Large-scale production is sometimes difficult because of high viscosity nature of cubosomes.

- There is a Low entrapment of water-soluble drugs due to the presence of high amounts of water inside of cubosomes.

- Cubosomes do not offer controlled drug delivery when they consist of polymer-based drug form.

- Cubosomes may lead to a problem of drug leakage in preparation, preservation and transport in vivo and may have a low drug loading efficiency; thus the major defect of their stability acts as a barrier and thus limiting their use. 


\section{Preparation methods of cubosomes}

The cubic lipid phases of cubosomes consists of three macroscopic forms that are typically encountered precursors, bulk phase gel and particulate dispersion. Bulk phase cubic gels that are rigid, strong, optically isotropic, and solid like particles which are in equilibrium with water and are dispersed into cubosome nanoparticles that has been made easier than their dispersions [26]. The nanoparticle dispersions prepared in the cubosomes can be done by several techniques such as spray drying, sonication, high pressure homogenization and spontaneous emulsification, whereas sonication and high-pressure homogenization and then forms the complex dispersions that contains vesicle like structures and cubosomes with time dependent ratios of each type of particle [27].

The production of cubosomes can be manufactured by two distinct technologies. They are:

\section{Top-down approach}

\section{Bottom-up approach}

\section{Top-down approach}

It is the most widely and frequently used procedure which was initially reported by Ljusberg-Wahren in 1996 [28]. This technology is carried out in two steps. The viscous bulk cubic phase is first produced by mixing the lipids with stabilizers; thus aggregation takes place. Second step is the bulk cubic phase is dispersed into aqueous medium to form Lyotropic Liquid Crystal (LLC) nanoparticles (cubosome dispersions) by the application of high energy processing such as high-pressure homogenization or sonication/high energy dispersions. A clear rigid gel formed by water-swollen cross-linked polymer chains which resembles the bulk cubic phases but they differ in that they are a single thermodynamic phase and have a periodic liquid crystalline structure $[28,29]$.

The high energy input process is required to homogenize the bulk phase is nearly not possible in the large scale, and it is very difficult to incorporate thermo-labile ingredients like peptides, and proteins as shown in fig. 4 [30]. Cubic Phases behave as lamellar phases during dispersion by increasing shear: vesicles form at intermediate shear rates, and a defect free bulk phase re-forms at higher shear rates, cubic phases become highly elastic at high oscillatory frequencies [26]. HPH is the most frequently used technique in the preparation of LLC nanoparticles. Cubosomes are prepared and observed to co-exist with vesicle-like structures [28].

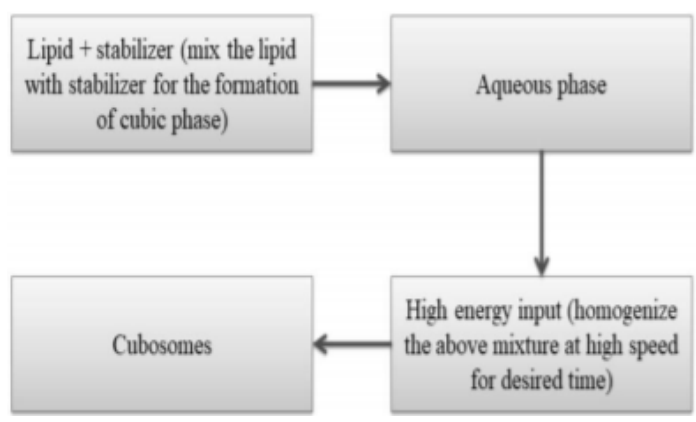

Fig. 4: Cubosomes by top down technique

\section{Bottom-up approach}

It is the most recently developed technique in the cubosome development, an alternative approach in the preparation of cubosomes at room temperature that allowing cubosomes to form crystallization from precursors on the molecular scale length. In this type of technique, it initially forms the building blocks in nanostructure and then assembles them into the final product [8, 30]. Scale up of this approach is found to be very difficult by the high process energy requirements to form the cubosome particle dispersions from the viscous bulk cubic phase. To avoid these problems Patric T. Spicer studied the formation of cubic phase in the presence of a hydrotrope. Hydrotrope here is a molecule that is either hydrophilic or hydrophobic but is incapable of exhibiting surfactant behaviour (Micelle formation). Hydrotropes do not produce LLC, but they increase the lipid solubility and then exhibit a phenomenon called "salting out" precursor may be either of a liquid or a solid. The liquid precursor is made by adding ethanol to the lipid (monoolein) ethanol. Cubosomes are produced, when the precursor is diluted. Powdered precursors comprise of a dehydrated substance that is coated with a polymer, forms cubosomes upon the hydration [31].

In this technique, the input factor hydrotrope gets dissolve in water insoluble lipids to create liquid-precursors. Powdered precursors have some advantage compared to liquid precursor cubosomes [32]. Less energy input is needed to produce cubosomes at high concentration to prevent the formation of liquid crystals when compared to top-down approach. The dispersion of inverse micellar phase droplets leads to the cubosomes formation in water at $80{ }^{\circ} \mathrm{C}$, and then allow them to slowly cool and droplets to gradually crystallize into cubosomes. Monoolein-ethanol mixture is then diluted with aqueous solution of poloxamer 407 which is produced from cubosomes at room temperature. Thus, cubosomes are formed by emulsification process as shown in fig. 5. This method cannot avoid the forming of vesicles through cryo-TEM, many vesicle sized-like structures are also observed to be coexist with cubosomes [23-29]. In the preparation of drug-containing vesicles used by freeze-drying technique. Freitas and Muller prepared solid lipid nanoparticles by using spray drying technology. Patrick T. Spicer was worked by using a spray drying technique on producing starchand dextran powder precursors, upon addition of water produce cubosomes. Thus, hydrophobically modified starch can be used to encapsulate monoolein. Starch-based monoolein-water system is then characterized by a "pseudo-ternary equilibrium phase diagram" [32].

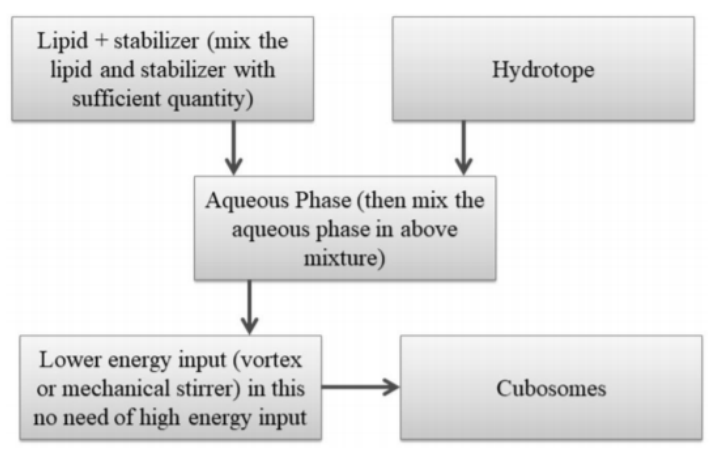

Fig. 5: Cubosomes by bottom up technique

The cubosome dispersion of the nanoparticles formed by several techniques such as sonication, high pressure homogenisation, spontaneous emulsification, spray drying and sonication and high pressure [8].

The cubosome dispersion is carried out by two methods:

\section{Fabrication method}

P407 cubic gel GMO 5\% or GMO and P407 1.0\% are melted at $60{ }^{\circ} \mathrm{C}$ in hot water bath and then add required quantity of drug and stir continuously till it dissolves. Deionized water is added drop by drop and vortex region is set to the homogenisation. It kept at room temperature for about $48 \mathrm{~h}$,forms the optically isotropic cubic gel and istotally distributed by the mechanical stirring [5]. Crude dispersion is normally fragmented by sonicater probe with the $200 \mathrm{~W}$ energy at the $20^{\circ} \mathrm{C}$ in water bath for about 20 min under cool temperature [25,33].

\section{Emulsification method}

In this process, GMO and P407 are added to water and then followed by the ultrasonication method; the 1\% P407, 5\% GMO and 5\% ethanol in $89 \%$ water are taken. GMO and P407 are melting at $60^{\circ}$ 
and then mixed with the ethanolic solution that was added to the melting. The resultant mixture is added dropwise to deionized water preheated at the $70{ }^{\circ} \mathrm{C}$, it ultrasonicated at the maximum power of $130 \mathrm{~kW}$ for about $50 \mathrm{~min}$ at the same temperature as the disperse mixture kept at the ambient temperature and protected from direct sunlight [25-34].

\section{Material used in cubosomes formation}

Bicontinuous cubic phases are to be found in natural lipids, although the lipids are most widely used to prepare bicontinuous cubic phases is the monoglyceride monoolein. Monoglycerides continuously form bicontinuous cubic phases of cubosomes particles, which are relatively insoluble upon the addition of water and are resistant to changes in temperature. The main precursor for the formation of cubosome is monoolein. Monoolein or glyceryl monooleate is a mixture of the glycerides of oleic acid and some other fatty acids, mainly consisting of the monooleate $[8,25]$. The glycerol moiety may form hydrogen bonds with water in an aqueous environment and is commonly termed as the head group. The hydrophobic characteristics from the hydrocarbon chain to monoolein and is often referred as the tail [8].

Monoolein, which is commercially available, are obtained in two forms, either as a mixed glyceride form or as distilled monoolein, which is mainly used for pharmaceutical applications because of its high purity. It swells in water, which gives several lyotropic liquid crystalline structures. When lipid molecule is heated instead of melting, it directly converts into an isotropic liquid. In the production of cubosomes, surfactant like poloxamer 407 is used in a concentration range between $0 \%$ to $20 \% \mathrm{w} / \mathrm{w}$, which are same as the disperse phase. The concentration of the monoglyceride or surfactant mixture generally takes place between $2.5 \%$ and $10 \%$ $\mathrm{w} / \mathrm{w}$ with respect to the total weight of the dispersion. Polyvinyl alcohol (PVA) is used in addition to poloxamer, as a stabilizing agent of the dispersion $[8,25]$.

\section{Evaluation of cubosomes [25]}

\section{Visual inspection}

The cubosomes are visually approved for the optical appearance like colour, turbidity, homogeneity and presence of macroscopic particles.

\section{Shape of the cubosome}

To view the shape of the cubosomes, Transmission electron microscopy can be used.

\section{Particle size distribution}

The cubosome nanoparticle size distribution are mainly determined by using Dynamic Laser Light Scattering by using Zeta sizer (Photon correlation spectroscopy). The sample is diluted with a suitable solvent and is adjusted to light scattering intensity of about $300 \mathrm{~Hz}$ and measured in triplicate at $25{ }^{\circ} \mathrm{C}$. That data is collected and generally shown by using average volume weight size. The zeta potential and polydispersity index are also recorded.

\section{Zeta potential}

The Zeta potential magnitude of cubosomes explains about the degree of electronic repulsion between the similarly charge particles. Zeta potential is a key indicator for the stability of formulation.

\section{Entrapment efficiency}

The entrapment efficiency of cubosomes is determined by using ultrafiltration techniques. The unentrapped drug concentration is also determined in the later technique that is subtracted from the total drug added. The amount of the drug is analysed by using spectrophotometer.

\section{Measurement of drug release}

The amount of drug release from cubosomes is measured by using pressure ultrafiltration method. The measurement of drug release is proposed by Magenheim, an Amicon pressure of ultrafiltration cell, then investigated on the fact which is fitted with a millipore membrane at ambient temperature of $(22 \pm 2){ }^{\circ} \mathrm{C}$.

\section{Stability studies}

It studied on the investigation of organoleptic and morphological studies of the physical stability as a function of time. Particle size distribution and drug content can be approached at different time intervals and can also be used to evaluate the possible variations by time.

\section{Applications of cubosomes \\ Controlled or sustained release of drugs}

Number of drugs having physicochemical properties are incorporated into cubosomes and their sustained behaviour of cubosomes, is because of cubosome remnant particles was also studied and used in skin and hair care, cosmetics and as antiperspirants. They are applicable as ophthalmic, transdermal and mucoadhesion [24, 25]. Controlled release of solubilised drug is the most popular application for cubosomes. Cubic lipid phases are more used for controlled release drugs because of their small pore size $(5-10 \mathrm{~nm})$, which have ability for solubilising hydrophilic, hydrophobic, amphiphilic molecules and by simple enzymes, biodegradability occurs [35].

\section{Melanoma (cancer) therapy}

In recent days, few anticancer drugs are spontaneously encapsulated into cubosomes and characterized the physicochemical properties. The distinctive structure of this promising nanocarrier suggests its application in this therapy. Different techniques have been investigated to specifically target nanomedicines to tumours regions in the body, with active and passive targeting of cancer cells in preclinical and clinical studies $[28,36]$.

\section{Cubosomes for the oral route}

Cubosome implements the various challenges in this delivery route of numerous promising materials that are having the high molecular size, poor absorption and poor aqueous solubility. In an alternative approach, large size proteins are encapsulated in the gastrointestinal region for the local activity. The cubosome nanoparticles are deliberated to form in-situ in a controlled rate manner, which facilitates an effective in vivo drug distribution. The technology of cubosome carriers provide the release of drugs at different absorption sites, for example, it is essential for the drugs to have narrow regional absorption window in the upper and lower intestinal regions [37].

\section{Cubosomes for the topical route}

As Cubic phases are having bio-adhesive properties, they are applicable in topical and mucosal surfaces and different delivery of drugs. These systems are based on the exploitation of distinct properties of liquid crystal and liquid crystal nanoparticle technologies. Topical drug delivery systems are unique in-situ, which form bio-adhesive LC systems that facilitate controlled and effective drug delivery to mucosal surfaces like buccal, ophthalmic, vaginal and others. This system forms a thin surface film at mucosal surfaces for achieving an optimal delivery profile and has excellent temporary protection for sore throat and sensitive skin [23].

\section{Cubosomes in intravenous route}

Cubosome cubic phase nanoparticles consists of interior liquid crystal nanostructures of lipid membranes that are used to solubilize, encapsulate and deliver nanomedicines to the affected regions in the body. Emulsions and liposomescan be used as intravenous carriers in drug formulations. Cubosomes particles of nanostructures show an increased number of peptides, proteins and many insoluble molecules and ideal carriers for injection [23, 25].

\section{Cubosomes in nasal route}

Direct nose-to-brain delivery of therapeutics, bypassing the bloodbrain barrier (BBB), provides a non-invasive and effective route in the treatment of central nervous system disorders. Gly14-human (S14G-HN) was incorporated into cubosomes and investigated its therapeutic effect in the AD. This result showed that using odorranalectin cubosomes can improve the effects of S14G-HN in AD. Mayuri Ahirrao, has investigated about the delivery of resveratrol targeting brain in nasal route delivery by cubosomes particles for treating Alzheimer's disease. GMO P407 cubosomes are 
made by probe sonication method. In vitro release of drug showed a controlled manner pattern for almost about $24 \mathrm{~h}$ [32].

\section{As a drug delivery vehicle}

The rapid growth of the life sciences industry is likely to be drive previously exotic delivery vehicles and ingredients into broader market places, such as personal care and consumer products. Consequently, self-assembled surfactant phases have been broadly examined for compatibility with more medical active ingredients and their applications. The cubosome nanoparticles are used in the form of oil-in-water emulsion stabilisers and absorbents in association with L'oreal and Nivea cosmetic companies [38].

\section{Cubosomes for ophthalmic drug delivery}

Drugs were administered to the eyes as drops because of the blinking, tears, nasolacrimal drainage drug removal is often. $\mathrm{pH}$, the lipophilicity of the drug and corneal epithelium are the main considerable facts that affect poor bioavailability of the drugs. In vitro corneal penetration evaluation proved their capability of increasing the transcorneal permeation of flubiprofen. Cubosomes are applied with dexamethasone to increase the pre-ocular retention and ocular bioavailability in the eye. The drug is incorporated into cubosomes exhibited about 3.5-and 1.8-fold increase in comparison with free dexamethasone eye drops [32, 39].

\section{In the treatment of viral diseases}

Cubosome nanoparticles can be applied in the design of intravaginal treatment of sexually transmitted diseases like HSV, HIV or by bacteria like Chlamydia trachomatis and Neisseria gonorrhoeae because of monoglycerides microbicidal properties [40].

\section{CONCLUSION}

Cubosomes are non-toxic, biocompatible, bioadhesive, have a larger surface area and are flexible to incorporate hydrophilic, hydrophobic or amphiphilic substances which have drawn the interest of the researchers to apply in drug delivery as carriers. Cubosome nanoparticles are lyotropic liquid crystalline materials that has found to be a potential advantage in drug delivery through various routes. Cubosomes have increased flexibility in product development because of their ability to produce cubosomes during production or during formulation. Cubosomes which are formed in dispersion possess a nanometer-scale structure which is identical to the bulk cubic phase. By using ultrasonication and high-pressure homogenization techniques, the cubosomes are produced by employing top-down and bottom-up approaches.

Cubosomes are applicable to a wide range of drug substances, proteins, immune substances and also to cosmetics. Cubosomes are novel surfactant-based carrier system which involves a simple preparation combination of biologically compatible lipids and water which are well suited for the skin, hair and other body tissues. The drugs are introduced into the pharma marketing in which half of the drugs containing protein and peptide forms and $80 \%$ of these proteins act as antibodies due to control release activity. Due to the potential site-specificity, the cubosomal formulatios could be widely employed as targeted drug delivery systems for ophthalmic, diabetic and also for anticancer therapy. Moreover, it has been reported that drug encapsulated cubosomes are applied as a controlled and sustained release drug carrier in recent studies.

\section{FUNDING}

None

\section{AUTHORS CONTRIBUTIONS}

All the authors have contributed equally.

\section{CONFLICT OF INTERESTS}

\section{Declared none}

\section{REFERENCES}

1. Andersson S, Jacob M, Larsson K. Structure of the cubosome- a closed lipid bilayer aggregate. Zeitschrift für Kristallographie.
Crystalline Materials. 1995;210(5):315-8. doi: 10.1524/ zkri.1995.210.5.315.

2. Spicer PT. Cubosomes bicontinuous cubic liquid crystalline nanostructured particles. Cincinnati: Procter and Gamble Company; 2004. doi: 10.1081/E-ENN, PMID 120014156.

3. Shah JC, Sadhale Y, Chilukuri DM. Cubic phase gels as drug delivery systems. Adv Drug Deliv Rev. 2001;47(2-3):229-50. doi: 10.1016/s0169-409x(01)00108-9, PMID 11311994.

4. Drummond CJ, Fong C. Surfactant self-assembly objects as novel drug delivery vehicles. Curr Opinion Colloid Interface Sci. 1999;4(6):449-56. doi: 10.1016/S1359-0294(00)00020-0.

5. Chong JYT, Drummond XMBBCJ. Steric stabilizers for cubic phase lyotropic liquid crystal nanodispersions (Cubosomes). Adv Planar Lipidic Bilayers Liposomes. 2015;21:131-87.

6. Larsson K, Fontell K, Krog N. Structural relationships between lamellar, cubic and hexagonal phases in monoglyceride-water systems. possibility of cubic structures in biological systems. Chem Phys Lipids. 1980;27(4):321-8. doi: 10.1016/00093084(80)90026-2.

7. Anbarasan B, Grace XF, Shanmuganathan S. An overview of cubosomes- smart drug delivery system. Sri Ramachandra J Med. 2015;8:1-4.

8. Khedekar PB. Cubosomes: a vehicle for delivery of various therapeutic agents. MOJ Toxicol. 2018;4(1):19-21. doi: 10.15406/mojt.2018.04.00083.

9. Karami Z, Hamidi M. Cubosomes: remarkable drug delivery potential. Drug Discov Today. 2016;21(5):789-801. doi: 10.1016/j.drudis.2016.01.004, PMID 26780385.

10. Urvi S, Dhiren D, Bhavin P. Overview of cubosomes: A nanoparticle. J Pharm Integr Life Sci. 2013;1(5):36-47.

11. Bouwstra JA, Honeywell Nguyen PL. Vesicles as a tool for transdermal and drug delivery system. Drug Discovery Today Drug Delivery Nanotech. 2005;2:67-74.

12. Daware SU, Saudagar RB. Formulation and development of cubosome loaded Emulgel-A review. Int J ChemTech Res. 2017;10:918-24.

13. Spicer PT, Hayden KL, Lynch ML, Ofori-Boateng A, Burns JL. Novel process for producing cubic liquid crystalline nanoparticles (Cubosomes). Langmuir. 2001;17(19):5748-56. doi: 10.1021/la010161w.

14. Almeida J, Edwards DC, Brand C, Heath T. Formation of virosomes from influenza subunits and liposomes. Lancet. 1975;306(7941):899-901. doi: 10.1016/S0140-6736(75)92130-3.

15. Makai M, Csanyi E, Dekany I, Nemeth Z, Eros I. Structural properties of nonionic surfactant/glycerol/paraffin lyotropic liquid crystals. Colloid Polym Sci. 2003;281(9):839-44. doi: 10.1007/s00396-002-0851-4.

16. Kulkarni CV, Wachter W, Iglesias Salto G, Engelskirchen S, Ahualli S. Monoolein: a magic lipid?. Phys Chem Chem Phys. 2011;13(8):3004-21. doi: 10.1039/C0CP01539C.

17. Montis C, Castroflorio B, Mendozza M, Salvatore A, Berti D, Baglioni P. Magneto cubosomes for the delivery and controlled release of therapeutics. J Colloid Interface Sci. 2015;449:31726. doi: 10.1016/j.jcis.2014.11.056, PMID 25533536.

18. Richert S, Schrader A, Schrader K. Transdermal delivery of two antioxidants from different cosmetic formulations. Int J Cosmet Sci. 2003;25(1-2):5-13. doi: 10.1046/j.14672494.2003.00158.x, PMID 18494876.

19. Boyd BJ, Whittaker DV, Khoo SM, Davey G. Lyotropic liquid crystalline phases formed from glycerate surfactants as sustained release drug delivery systems. Int J Pharm. 2006;309(1-2):218-26. doi: 10.1016/j.ijpharm.2005.11.033, PMID 16413980.

20. Chong JYT, Mulet X, Boyd BJ, Drummond CJ. Steric stabilizers for cubic phase lyotropic liquid crystal nanodispersions (cubosomes). Adv Planar Lipid Bilayers Liposomes. 2015;21:131-87. doi: 10.1016/bs.adplan.2014.11.001.

21. Wadsten Hindrichsen P, Bender J, Unga J, Engström S. Aqueous self-assembly of phytantriol in ternary systems: effect of monoolein, distearoylphosphatidylglycerol and three watermiscible solvents. J Colloid Interface Sci. 2007;315(2):701-13. doi: 10.1016/j.jcis.2007.07.011, PMID 17655855.

22. Zhai J, Waddington L, Wooster TJ, Aguilar MI, Boyd BJ. Revisiting beta-casein as a stabilizer for lipid liquid crystalline 
nanostructured particles. Langmuir. 2011;27(24):14757-66. doi: 10.1021/la203061f, PMID 22026367.

23. Dhadwal A, Sharma DR, Pandit V, Ashawat MS, Kumar P. Cubosomes: A novel carrier for transdermal drug delivery. J Drug Delivery Ther. 2020;10(1):123-30. doi: 10.22270/jddt.v10i1.3814.

24. Naveentaj S, Muzib YI. A review on liquid crystalline nanoparticles (cubosomes): emerging nanoparticulate drug carrier. Int J Curr Pharm Sci. 2020;12(1):5-9. doi: 10.22159/ijcpr.2020v12i1.36820.

25. Sadhu VR, Beram NS, Kantamneni P. A review on cubosome: the novel drug delivery system. GSC Biol Pharm Sci. 2018;5(1):76-81. doi: 10.30574/gscbps.2018.5.1.0089.

26. Radiman S, Toprakcioglu C, Mcleish T. Rheological study of ternary cubic phases. Langmuir. 1994;10(1):61-7. doi: 10.1021/la00013a009.

27. Almgren $\mathrm{M}$, Edwards $\mathrm{K}$, Gustafsson J. Cryotransmission electron microscopy of thin vitrified samples. Curr Opin Colloid Interface Sci. 1996;1(2):270-8. doi: 10.1016/S1359. 0294(96)80015-X.

28. Bei D, Meng J, Youan BB. Engineering nanomedicines for improved melanoma therapy: progress and promises. Nanomedicine (Lond). 2010;5(9):1385-99. doi: 10.2217/nnm.10.117, PMID 21128721.

29. Spicer PT, Small WB, Small WB, Lynch ML, Burns JL. Dry Powder Precursors of Cubic Liquid Crystalline Nanoparticles (cubosomes). J Nanoparticle Res. 2002;4(4):297-311. doi: 10.1023/A:1021184216308.

30. Worle G, Drechsler M, Koch MH, Siekmann B, Westesen K, Bunjes $\mathrm{H}$. Influence of composition and preparation parameters on the properties of aqueous monoolein dispersions. Int Pharm. 2007;329(1-2):150-7. doi: 10.1016/ j.ijpharm.2006.08.023, PMID 16987623.
31. Khalifa MK. Miconazole nitrate based cubsosome hydrogels for topical application. Int J Drug Deliv. 2015;7:1-12.

32. Molly BA, Prasanthi NL. Cubic liquid crystalline nanoparticles (Cubosomes): A novel carrier for drug delivery. Int J Pharm Sci Res. 2019;10(3):973-84.

33. Bhowmik D, Gopinath H, Kumar BP. Recent advances in novel topical drug delivery system. J Pharm Innov. 2012;1(9):12.

34. Thorat YS, Gonjari ID, Hosmani AH. Solubility enhancement techniques: a review on conventional and novel approaches. Int J Pharm Sci Res. 2011;2(10):2501.

35. Nilsson C, Ostergaard J, Larsen SW, Larsen C, Urtti A, Yaghmur A. PEGylation of phytantriol-based lyotropic liquid crystalline particles--the effect of lipid composition, PEG chain length, and temperature on the internal nanostructure. Langmuir. 2014;30(22):6398-407. doi: 10.1021/la501411w, PMID 24833115.

36. Sagnella S, Drummond C. Drug delivery a nanomedicine approach. Aust Biochem. 2012;43:5-7.

37. Elnaggar YS, Etman SM, Abdelmonsif DA, Abdallah OY. Novel piperine-loaded tween-integrated monoolein cubosomes as braintargeted oral nanomedicine in Alzheimer's disease: pharmaceutical, biological, and toxicological studies. Int J Nanomed. 2015;10:5459-73. doi: 10.2147/IJN.S87336, PMID 26346130.

38. Afriat I, Biatry B. Use of cubic gel particles as agents against pollutants, especially in a cosmetic composition. EP Eur Pat Appl (L'Oreal, Fr.); 2001.

39. Gan L, Han S, Shen J, Zhu J, Zhu C, Zhang X, Gan Y. Self-assembled liquid crystalline nanoparticles as a novel ophthalmic delivery system for dexamethasone: improving preocular retention and ocular bioavailability. Int J Pharm. 2010;396(1-2):179-87. doi: 10.1016/j.ijpharm.2010.06.015, PMID 20558263.

40. Thadanki M, Kumari PS, Prabha KS. Overview of cubosomes: a nanoparticle. Int J Res Pharm Chem. 2011;1(3):535-41. 